\title{
Síndrome de Burnout en el personal docente de enfermería
}

\author{
Ihosvany Basset Machado,* Rafael Antonio Estévez Ramos*
}

\begin{abstract}
RESUMEN
El síndrome de Burnout ha sido definido como una respuesta a la estresante situación laboral crónica que se produce, principalmente, en el marco del mercado laboral de las profesiones que se centran en la prestación de servicios, no escapando de esta situación aquellos profesionales de la salud y, en específico, el personal docente que prepara continuamente a las futuras generaciones de la salud en escenarios educativos. El objetivo de estas profesiones es cuidar los intereses o satisfacer las necesidades del estudiante en cualquiera de los niveles docentes; también se caracterizan por el contacto directo con los alumnos. El presente artículo tiene como objetivo realizar una investigación documental acerca del índice del síndrome de Burnout en el personal docente. Se utilizó, para el conocimiento del mismo, la revisión de artículos relacionados con el tema.
\end{abstract}

Palabras claves: Burnout, síndrome, docente, enfermería.

\section{Burnout syndrome among nursing teaching personnel}

\begin{abstract}
The syndrome of Burnout has been defined as a response to the stressful chronic labor situation that takes place, mainly, within the framework of the labor market of the professions that are centered in the benefit of services, not saving of this situation those professionals of the specific health and in the educational personnel who prepares continuously to the future generations of the health in educative scenes. The objective of these professions is to take care of the interests or to satisfy the necessities with the student in anyone of the educational levels, characterizing itself by the direct bonding with the students. The present article must like objective make a documentary investigation about the index of the syndrome of Burnout in the educational personnel. The article revision was used for the knowledge of the himself related to the subject.
\end{abstract}

Key words: Burnout, syndrome, educational, nursing.

Introducción

$\mathrm{E}$ 1 síndrome de Burnout se presenta con relativa frecuencia en el personal que labora en instituciones educativas o de salud como una respuesta psicoso- cial afectiva por encontrarse emocionalmente agotado; se manifiesta por actitudes y sentimientos negativos hacia las personas con las que se trabaja.

Diversos autores han documentado que este síndrome afecta más a aquellas profesiones que requieren un contacto directo

* Doctor en Ciencias de la Salud, Universidad Autónoma del Estado de México, Centro Universitario Valle de Chalco, Secretaría de Salud. Instituto de Salud del Estado de México.

Consejo Nacional de Ciencia y Tecnología. Dirección Adjunta de Desarrollo Científico. Dirección de Planeación de Ciencia CONACyT.

Correspondencia: Dr. en C. S. Ihosvany Basset Machado. Universidad Autónoma del Estado de México, Centro Universitario Valle de Chalco, Secretaría de Salud. Instituto de Salud del Estado de México. Tel: 309217 63. Ext: 205, Cel. 552330730958601907. E-mail: ibassetcuba@ hotmail.com.mx

Este artículo puede ser consultado en versión completa en http://www.medigraphic.com/enfermerianeurologica 
con las personas y con una filosofía humanística del trabajo, es decir, aquellas que necesitan altas dosis de entrega e implicación. Así, son muchos los profesionales potencialmente afectados por el síndrome (docentes, enfermeros, médicos, trabajadores sociales, profesores, policías, entre otras profesiones u oficios), principalmente aquéllos cuyo trabajo tiene repercusión directa sobre la vida de otras personas; entre éstos se encuentra el personal docente, por ser una de las profesiones que exige entrega, idealismo y servicio a los demás; se cimienta sobre una personalidad perfeccionista, o con un alto grado de autoexigencia, comprometiéndose en el trabajo de la enseñanza; esto puede causarle diversos síntomas como: falta de ilusión, desmotivación por el trabajo, agotamiento físico y psíquico, bajo rendimiento laboral, frustración, etcétera (Maslach, 1998).

El estrés forma parte de nuestra vida cotidiana, hasta tal punto que puede considerarse como el malestar de nuestra civilización; afecta tanto a la salud y al bienestar personal como a la satisfacción laboral y colectiva. En el contexto de la salud laboral surge el desarrollo de un nuevo proceso: el síndrome de Burnout (Gil-Monte, 1997).

Para otros autores, el síndrome de Burnout se refiere a un proceso en el que los profesionales están agotados de su trabajo, en respuesta a las experiencias de estrés y tensión en el mismo. Los problemas que pueden ser solventados no llevan al Burnout, de hecho el estrés controlado puede tener efectos positivos, estimulando al individuo a superarse, lo que culminaría en una experiencia gratificante y en competencia profesional. Precisamente, el Burnout comienza cuando el individuo se siente más indefenso para cambiar la situación. Otros factores sobre los que existe más controversia son los diferentes turnos de trabajo que implican en algunos profesionistas, trabajar en otras instituciones; frente a este escenario existe mayor compromiso. De la misma manera, los horarios laborales, el salario, la categoría profesional y la sobrecarga laboral son algunos de estos factores que pueden estar implicados en desarrollar mayor riesgo de presentar estrés o el propio síndrome.

Además, siguiendo la bibliografía existente, se detectan una serie de áreas de trabajo con mayor incidencia del síndrome, como es el caso de las instituciones educativas y hospitalarias.

Al considerar los factores institucionales, diferentes autores destacan positivamente el soporte social como el principal medio para contrarrestar los efectos del Burnout. Por el contrario, se han identificado numerosos factores estresantes crónicos del entorno laboral implicados en su producción, como los estudiantes difíciles que presentan seguidamente su propio inconformismo; el poco tiempo de atención por estudiantes y la mala calidad de las relaciones del equipo de docentes o docente/estudiante (Atance, 1997).
El personal que labora en las instituciones de educación no escapa de la posibilidad de presentar este tipo de síndrome; el estrés ocasionado por la atención diferente y particularizada que se debe brindar a una gran cantidad de estudiantes suele ser de mayor complejidad, aunado a la escasez de recursos materiales para ofrecer la atención requerida, asî como los factores externos al medio laboral que incide en los profesionales que se dedican a esta ocupación: la docencia. De primera instancia y para poder desarrollar el artículo de revisión, sólo se abordaron algunos estudios realizados en este medio profesional para los efectos de la investigación directamente y así poder tener un acercamiento a este síndrome en el personal docente.

En la década de los setenta, a consecuencia de los estudios que se realizaron sobre el estrés, se presenta el concepto Burnout en las primeras investigaciones de Freudenberger (1974). Aunque antes de finalizar esta década son muchas las referencias sobre este tema en diversas profesiones asistenciales, entre ellas la de los docentes Keavney y Sinclair (1978) y Kyriacou y Sutcliffe (1977), no será hasta los ochenta cuando se produzca un aumento considerable de las investigaciones sobre Burnout.

El concepto de estrés ha sido muy polémico dentro de la psicología, tanto en su definición como en sus modelos teóricos explicativos (Sandín, 2003). Sin embargo, el término Burnout ha sido delimitado y aceptado por la comunidad científica casi en su totalidad desde la conceptualización establecida por Maslach (Maslach, 1982, 1999) en la que define el Burnout como una respuesta del estrés crónico formada por tres factores fundamentales: cansancio emocional, despersonalización y baja realización personal.

Los primeros detonantes que comenzaron a señalar la importancia del estrés y Burnout en la profesión docente fueron los datos que las diferentes administraciones de educación tenían sobre las bajas laborales de los profesores y su evolución a través de los años. Estos resultados señalaban un aumento anual de las bajas de tipo psiquiátrico que no se encontraban en otras profesiones y que tenían como consecuencia un incremento del absentismo laboral, un enorme gasto en sustituciones y un bajo rendimiento en el trabajo (García-Calleja, 1991).

Al respecto, se hace mención de algunas de las características que se pueden dar de una manera generalizada en las investigaciones sobre el estrés y Burnout:

1. La utilización de estrategias correlacionales y ausencia de las experimentales.

2. El análisis de numerosas variables sociodemográficas, de la personalidad y de la organización, buscando correlaciones de éstas con el Burnout. 
3. Los estudios epidemiológicos son meramente descriptivos del número de bajas, tipo, duración, etcétera, durante un determinado número de cursos académicos.

4. El elevado número de resultados contradictorios exige plantear la posibilidad de que los procedimientos metodológicos utilizados sean muy flexibles y que la fiabilidad y validez de estas investigaciones puedan verse afectadas por las dificultades de definir muchos de los términos utilizados.

Existen estudios descriptivos con diseños correlacionales que estudian la relación entre el estrés, Burnout y otras variables fundamentalmente sociodemográficas que se repiten en casi todos los países. En Alemania, Knight-Wegenstein (1973) realizó uno de los estudios pioneros en el ámbito de la educación en el que obtuvo que un $87.6 \%$ de los 9,129 profesores estudiados aparecieran como afectados por un elevado grado de tensión en el trabajo.

En Alemania, Kohnen y Barth (1990) realizaron un estudio en una población de 122 docentes, en el cual se refiere que sólo el $28 \%$ comunicaba síntomas mínimos sobre Burnout frente a un $43 \%$ con síntomas moderados y un $28.7 \%$ con síntomas más severos. Se destacan también aquéllas realizadas por Esteve (1994) en España; Pines, Aroson y Kafry (1981) en Estados Unidos; Borg y Falzon (1989) en Malta; Brenner (1982) en Suecia, Kyriacou (1980), Capel (1987), Chakravorty (1989) en Inglaterra y Temml (1994) en Austria.

Para la investigación es importante analizar los estudios que buscan una relación entre las bajas laborales, el estrés y Burnout. Chakravorty (1989) realizó una investigación sobre el tema donde hace referencia que un $77 \%$ de las bajas de larga duración era debido a trastornos mentales en una muestra de 1,500 docentes con baja laboral.

En otros estudios, relacionan puntuaciones de medidas en el síndrome de Burnout (siendo la más utilizada el MBI) siempre con variables sociodemográficas (sexo, edad, etcétera) en diferentes profesiones, realizando estudios comparativos directamente con docentes (de Heus y Diekstra, 1999; Travers y Cooper, 1997). Las profesiones más estudiadas en este campo han sido el personal profesional y no profesional de enfermería: médicos, trabajadoras sociales, rehabilitadores físicos, trabajadores de la salud mental, entre otros. En todos estos estudios se expresa que la profesión más afectada por el síndrome de Burnout es el personal docente, que en su gran mayoría está relacionado directamente con el trabajo en escenarios hospitalarios y con la profesión docente en áreas de educación (escuelas).

Se revisaron algunas investigaciones que estuvieran relacionadas con las consecuencias, variables asociadas y causas del estrés y el Burnout. En docentes se analizaron modelos teóricos y explicativos de los más citados en la literatura científica sobre este tema, así como los diversos sistemas de evaluación y medidas de estrés y Burnout más utilizados, recopilando los diferentes tipos de técnicas de intervención empleadas para tener conocimiento sobre las principales medidas que deberían aportar las instituciones educativas para paliar sus efectos organizativos en el estrés y el Burnout a nivel individual y grupal, así como su prevención.

\section{CAUSAS Y VARIABLES RELACIONADAS CON EL ESTRÉS Y EL BURNOUT EN PROFESORES}

Las variables son un tema de gran interés, ya que éstas están directamente relacionadas con la etiología de este fenómeno: hoy en día el estrés y Burnout en docentes.

En este sentido, se describen las más estudiadas y las principales conclusiones a las que se han llegado. Distinguiéndose entre las variables personales sociodemográficas, como la edad, el sexo y las variables personales relacionadas con la personalidad y actitud del sujeto en estudio.

\section{VARIABLES SOCIODEMOGRÁFICAS}

Son muchos los estudios (Byrne, 1991; Maslach y Jackson, 1981; Schaw e Iwanicki, 1982) donde su atención central es el análisis de las variables demográficas y la relación existente entre el estrés y el Burnout. En la gran mayoría de estos estudios se puede observar, mediante el análisis, bajos índices de porcentajes significativos de varianza en diversas subescalas que evalúan el Burnout. Sin embargo, en una parte importante de las variables se observan resultados que pudieran ser, bajo el análisis, contradictorios, y esta contradicción está presente entre los diferentes estudios epidemiológicos correlacionales con Burnout. Se puede encontrar que, por ejemplo, las investigaciones en pocas ocasiones son longitudinales o experimentales.

\section{Sexo}

Maslach (1982) afirma que las mujeres pueden experimentar de forma más intensa el cansancio emocional en comparación con los hombres, pero Schwab e Iwanicki (1982) y Gil-Monte y Peiró (1997) encuentran mayor varianza en la despersonalización; de acuerdo con el resultado final del estudio, los hombres son los que tienen mayor sentimiento negativo hacia los alumnos. Estos primeros resultados se contradicen con los estudios de Maslach y Jackson (1981), donde exponen diferencias en un mayor grado de cansancio emocional y menor realización personal en mujeres. Sin embargo, otros autores como Abraham (1984) y Maslach (1999) centran su atención en el doble rol que ejercen las mujeres en el trabajo y en la casa como fuente de estrés. 
Pero sin tener dudas al respecto, continúan siendo mayoría los autores que no encuentran demostración suficiente de diferencias significativas con la variable en cuestión: sexo (Byrne, 1999; Schwab e Iwanicki, 1982).

\section{Edad}

También se localizaron resultados contradictorios en esta variable, ya que la relación establecida entre la edad y el Burnout ha sido un tanto lineal (Seltzer y Numerof, 1988) como curvilínea; en el estudio de Golembiewski, Munzenriper, Stevenson (1986) y Whitehead (1986) también se encontró ausencia de relación (Hock, 1988). Estos resultados deberían ser matizados ya que la relación que se establece entre el síndrome de Burnout y la edad se asocia al tiempo de experiencia en la profesión, la maduración propia por la edad del individuo y con la pérdida de una visión irreal de la vida en general. Se pone de ejemplo en estos estudios que los docentes más jóvenes presentan niveles superiores al estrés; esto puede entenderse, precisamente, por la falta de experiencia laboral en este medio, además de estar en edades relativamente homólogas a la de los estudiantes. Otro ejemplo que los autores Yagil (1998), Crane e Iwanicki (1986), y Schawb e Iwanicki (1982) refieren es que los docentes más jóvenes experimentan niveles superiores de estrés, mayores niveles de cansancio emocional y fatiga.

Malik, Mueller y Meinke (1991) no refieren diferencias significativas respecto a la edad. Sin embargo, Van Ginkel (1987) y Borg y Falzon (1989) refieren que aquellos docentes más experimentados, que llevaban más de veinte años en la profesión, tenían una respuesta de estrés mayor que la de sus compañeros con menos antigüedad.

\section{Tipo de centro educativo}

Los centros en donde se desarrolla la función docente tienen grandes implicaciones en la percepción de estrés por parte de los docentes. Si diferenciamos éstos por encontrarse en núcleos urbanos, rurales o por ser centros denominados suburbanos (tradicionalmente denominados marginales), todas las investigaciones apuntan en la misma dirección: existen mayores índices de Burnout en los centros suburbanos que en los dos anteriores (Byrne, 1999; Leithwood, Jantzi y Steinbach, 2001; Valero, 1997). Parece ser que el comportamiento de los alumnos sería el factor principal, ya que se trata de personas que frecuentemente pertenecen a minorías marginadas y a familias disfuncionales.

\section{Nivel impartido}

Se ha podido experimentar que, según vayamos avanzando en los ciclos o grados educativos, el índice de Burnout se puede incrementar. Al respecto, los docentes de nivel de secundaria básica son los más afectados (según Beer y Beer, 1992; Burke y Greenglass 1989), específicamente cuando nos referimos a las subescalas de despersonalización y realización personal (Anderson e Iwanicki, 1984). Gold y Grant (1993) explicaron estos resultados en secundaria, dado que los estudiantes presentan un menor grado de interés y motivación. Otros autores como Doménech (1995) y Ortiz (1995) señalaron un mayor nivel de Burnout en los primeros ciclos educativos. En un estudio minucioso en este tipo de investigaciones, se corroboró que el grupo más afectado es el de nivel de secundaria. Diversos autores (Anderson e Iwanicki, 1984; Beer y Beer, 1992) concuerdan en los problemas de conducta de jóvenes y adolescentes, y es donde se plantea el gran dilema de la educación obligatoria con sus respectivos límites de edad, el fallo de los sistemas de disciplina y los enfrentamientos de los alumnos con los docentes de este nivel educativo; además, tomando en cuenta todas aquellas implicaciones jurídicas que pueden conllevar según se encuentre el escenario.

\section{Estado civil y las relaciones familiares}

El estado civil y las relaciones familiares han sido de las características más estudiadas. Los docentes que presentan un estado civil de soltería, experimentan mayor grado de Burnout que los casados (Golembiewskiet et al., 1986), de manera que presentan un mayor cansancio emocional y despersonalización en el trato; sin embargo, estas condiciones no siempre se reproducen de modo exacto, ya que existen estudios donde los resultados son distintos. Durán, Extremera y Rey (2001) refieren que la paternidad puede funcionar como un factor protector ante el síndrome de Burnout, ya que se relaciona con la maduración que acompaña el ser padre, con una mayor experiencia en resolver problemas en los que pueden estar involucrados los hijos, así como el apoyo emocional recibido por parte de la familia. Desde este punto de vista, los autores consideran que llevar a casa aspectos relacionados con el trabajo puede ser negativo para las relaciones interpersonales del docente dentro del seno familiar y también una considerable fuente de estrés. Por otro lado, Cooke y Rousseau (1984) señalan que en algunos casos esta situación puede tener un impacto positivo y propositivo, y puede, incluso, propiciar una oportunidad para recibir apoyo, un consejo o también puede ser una forma de desahogarse, en la cual la familia podría incluir ideas que tal vez no se le habían ocurrido al docente; finalmente, sentirá el apoyo directo de la familia y, por ende, satisfacción en la toma de decisiones que repercutirá en el buen desempeño profesional de este docente. 


\section{VARIABLES DE PERSONALIDAD}

Cuando nos referimos a personalidad, nos enfrentamos a diversos tipos y variables con características indistintas del personal docente relacionadas con el estrés y el síndrome de Burnout.

Respecto a las variables estudiadas, el patrón de conducta tipo A (PCTA) tradicionalmente ha sido asociado al Burnout como modulador o facilitador de la experiencia de estrés. En el ámbito educativo, el síndrome ha estado asociado con la aparición de trastornos neuróticos y somáticos causados por el estrés que no presentan docentes ajenos a este tipo de conducta, obteniéndose una correlación significativa entre el Burnout y el PCTA. Sin embargo, no encontraban relaciones significativas entre este patrón de conducta y la experiencia de estrés. Por otro lado, recientes investigaciones señalan una elevada relación entre el PCTA, el Burnout y los problemas de salud mental de docentes con baja laboral de tipo psiquiátrico en comparación con docentes sin baja laboral, señalando a este patrón de conducta como un factor de riesgo de padecer este tipo de baja (Moriana, 2002).

La autoestima del docente es otra de las características asociadas o relacionadas con el síndrome de Burnout. En la mayoría de estudios aparece una correlación significativa entre docentes que presentaron baja autoestima y, por ende, mayores niveles del síndrome pudiendo llegar a la conclusión, en este aspecto, de que a medida que la autoestima disminuye en el docente, avanza el síndrome de Burnout. Cuando hacemos referencia al locus de control, gran parte de las investigaciones científicas apuntan a que los docentes con más locus de control externo tienen mayor tendencia de padecer síndrome de Burnout; indicando incluso una correlación de significancia entre las escalas de Burnout MBI y el locus de control (McIntyre, 1981).

En las investigaciones que se han realizado, la autoconciencia, el autocontrol y la autoeficacia ha dado en sus resultados que a mayor autoconciencia, mayor nivel de autocontrol y autoeficacia; esto supone un factor protector ante el síndrome. Ahora bien, cuando existen niveles altos del síndrome de Burnout, éstos se correlacionan con puntuaciones bajas en estas variables. Pines (1993) hace referencia a que sólo por el hecho de que las personas estén muy motivadas y con alta autoeficacia corren el riesgo de padecer Burnout, experimentando sólo estrés aquellas que se encuentran poco motivadas y con baja autoeficacia, fatiga e insatisfacción.

Las variables como el neuroticismo, la falta de empatía y los pensamientos irracionales también han sido relacionadas por algunos autores con el aumento de los índices de estrés y Burnout en los docentes.

\section{CICLOS DE ESTRÉS DEL DOCENTE}

Los docentes enfrentan características propias de la institución con la gran diversidad de actividades a lo largo del semestre o del ciclo académico; es importante señalar que durante el transcurso del ciclo, el docente enfrenta momentos en los que se puede encontrar más estresado. La incorporación al trabajo después de las vacaciones, los periodos de exámenes, etcétera, hacen del trabajo docente una situación dinámica en la que no siempre se realiza el mismo trabajo y en las que la época del curso puede predeterminar momentos vivenciados de forma muy dispar entre los docentes. En este sentido, en un estudio realizado en Estados Unidos de Norte América, en Nueva York en 1980, se hace referencia que la primera semana del curso escolar es la más estresante del año (Travers y Cooper, 1994).

\section{¿CUÁLES SERÍAN LAS CONSECUENCIAS DEL ESTRÉS Y EL BURNOUT EN LOS DOCENTES?}

Matud y García (2002) señalan que la insatisfacción con el rol laboral y la presión en el trabajo correlacionan con la sintomatología de tipo somático, depresiva, de ansiedad e insomnio. Calvete y Villa (2000) presentan coeficientes de correlación significativos entre el cansancio emocional y síntomas de depresión, somatización, ansiedad, dificultades cognitivas y sensibilidad interpersonal. Pines et al. (1981) señalan que las consecuencias que afectan al profesor que padece Burnout a nivel psicológico son numerosas: falta de autorrealización, baja autoestima, aislamiento, tendencia a la autoculpa, actitudes negativas hacia uno mismo y hacia los demás, sentimientos de inferioridad y de incompetencia, pérdida de ideales, irritabilidad y hasta casos más extremos de intentos de suicidio, cuadros depresivos graves, ansiedad generalizada, fobia social y agorafobia.

Se hace mención que, como consecuencia del estrés y Burnout, la insatisfacción laboral es alta, citada por un alto número de autores (Kyriacou y Sutcliffe, 1978; Travers y Cooper, 1997); las consecuencias que el estrés y el Burnout tienen sobre la familia han sido poco estudiadas en la literatura, convencidos de que debe ser un campo de estudio que se debe explotar por la significancia que puede arrojar. Se describen profesores que llegan tensos a casa, agotados física y psicológicamente, con cuadros de irritación, cansados de escuchar y hablar sobre problemas de otras personas. Estas condiciones no propician en absoluto un clima ideal para una adecuada vida familiar y de pareja. La vida de la pareja sufre un enorme deterioro, siendo la responsable de un importante número de divorcios dentro de este tipo de profesiones (Cooke y Rousseau, 1984). 


\section{INTERVENCIÓN, TRATAMIENTO Y PREVENCIÓN DEL BURNOUT EN PROFESORES}

Teniendo en cuenta el entorno laboral, las intervenciones en el síndrome de Burnout se deberán trabajar a nivel de organización, a nivel interpersonal (entre nuestros compañeros) y a nivel individual. Todos ellos deberán ser programados por la empresa ya que son de tipo organizacional; algunas técnicas que se pueden utilizar, como el trabajo con técnicas de reestructuración cognitiva, consistirían en tomar las situaciones problemáticas y volver a evaluarlas y reestructurarlas para que dejen de ser problemáticas.

También es útil que nos adiestremos en técnicas de autorregulación o de control, de gestión del tiempo, de delegación, desarrollo en habilidades sociales, asertividad y de entrenamiento en solución de problemas. Y todo esto, para conseguir que podamos mantener la motivación y el sentido que le dábamos a nuestro trabajo.

El aprendizaje de técnicas de relajación, yoga o cualquier otra técnica que nos permita estar en unas buenas condiciones para poder afrontar mecanismos estresantes. Algunos autores sugieren que de este modo será difícil que presentemos algún síntoma del Burnout, ansiedad o de estrés en el trabajo.

La intervención se debe realizar cuando el síndrome de estar quemado hace estragos en las personas. Muchas veces, los primeros en darse cuenta de la presencia del Burnout son las personas que nos rodean; es muy difícil que si estamos afectados por este síndrome, nos demos cuenta de ello, sobre todo cuando ya está muy avanzado. Es bueno en estos momentos que recibamos el apoyo de nuestros compañeros (apoyo social). Una buena calidad de relaciones interpersonales en el trabajo modera el nivel de Burnout, juega un importante papel en la satisfacción laboral y aumenta la calidad de vida en el trabajo. La comunicación tiene un papel muy importante (Gold, 1984).

Como el Burnout afecta a muchas áreas de la vida, se debe utilizar un modelo de intervención de varios componentes integrados, de forma que se complementen unos a otros. A nivel individual, se trata de mejorar los recursos de protección o de resistencia para afrontar de forma más eficiente los estresores típicos de la función laboral y potenciar la percepción de control, la autoeficacia personal y la autoestima. Normalmente se han utilizado intervenciones de tipo cognitivo-conductual, como, por ejemplo, las del afrontamiento del estrés, reestructuración cognitiva, terapia racional emotiva y ensayo conductual. Desde la perspectiva cognitiva es esencial hacer frente a las cogniciones erróneas o desadaptativas, como expectativas irreales y falsas esperanzas. Las medidas de prevención se pueden clasificar en tres apartados según sea la forma de aplicación: estrategias de intervenciones individuales, grupales y organizacionales. Esta situación implica una baja actuación por parte de los poderes públicos en la prevención de este fenómeno. De manera mayoritaria está considerada como la actuación más importante según la literatura sobre el tema. Siguiendo las líneas de actuación propuestas por Kelchtermans y Strittmatter (1999) para la prevención del Burnout en docentes, es necesario una actuación de base, desde la administración pública centrada en el reconocimiento público de la labor docente, la vuelta a una imagen positiva del profesor, el balance entre las políticas educativas y la realidad, así como la autonomía de los centros. Otros aspectos destacados son la provisión de recursos personales y materiales para poder adaptarse a los cambios que generan las necesidades sociales y políticas, analizar la carrera profesional y vida laboral del docente (remuneración económica, posibilidad de promoción, etcétera) y revisar las funciones de la escuela y de los docentes. También destacan la participación de éstos en las decisiones de los centros y en las políticas educativas, potenciar el trabajo en grupo, así como mantener adecuadas relaciones interpersonales entre los compañeros y crear servicios de apoyo y asesoramiento a la plantilla docente.

\section{CONCLUSIONES}

Luego de revisar algunas publicaciones o trabajos de investigación sobre el estrés y Burnout en el personal docente podemos constatar las múltiples contradicciones que surgen en algunos de los temas más estudiados, principalmente lo relacionado con las causas.

Existen limitaciones sobre la metodología ya que se puede observar la falta de acuerdos entre los diferentes autores. Se observan dificultades en poder manipular las variables dentro de un contexto educativo; el control de factores ambientales está propiciando que la mayoría de investigaciones sea de corte correlacional.

Los estudios pilotos deben potenciarse en los centros en donde se pueda posibilitar la variación de algunas características donde se arroja más información sobre los efectos de las variables del contexto que permitan integrar y asumir estrategias de tipo experimental en la investigación.

Dentro de las causas que afectan el malestar docente se destacan las siguientes variables: la sobrecarga laboral, el comportamiento de los alumnos en los escenarios escolares, los conflictos interpersonales con otros docentes, superiores y problemas derivados de las políticas educativas.

Es recomendable que las investigaciones se encaminen a estudiar a profundidad las variables de la personalidad, lo anterior por la razón de que se deben incluir marcadores que señalen de manera predictiva la vulnerabilidad del docente a padecer estrés y, por ende, a desarrollar Burnout o, en su 
defecto, patologías psicológicas características de cuadros que están relacionados con la ansiedad y la depresión, pudiendo convertirse en líneas de investigaciones a futuro, permitiéndonos conocer de manera más exacta y amplia el deterioro progresivo que puede sufrir el personal docente en su centro de trabajo y, en específico, en el puesto que desarrolla laboralmente.

Se debe enfatizar en la importancia que tiene el detectar de forma inmediata los estados iniciales del estrés y Burnout, ya que éstos pueden estar relacionados con el absentismo, bajas de tipo laboral por situaciones psiquiátricas, estados de conductas inadecuadas, despersonalización en el trato con los estudiantes, apatía, cansancio laboral, etcétera.

De manera final, se recomienda enfatizar específicamente en la detección precoz de los estados iniciales de estrés y Burnout que están íntimamente relacionados con el absentismo, bajas laborales de tipo psiquiátrico. Esto potenciaría una mejor salud mental del docente y una disminución del gasto personal y económico que en la actualidad padece gran parte de las instituciones de educación en el país.

En las diversas investigaciones realizadas y revisadas sobre el tema, siempre hacemos referencia a Maslach, teniendo la percepción que la definición sobre evaluación, modelo teórico y evolución del Burnout que ha aportado, es actualmente la más aceptada por la mayoría de investigadores, según la literatura. Esto posibilita una mejor delimitación conceptual de nuestro objeto de estudio y una mejora en los planteamientos metodológicos aplicados, no permitiendo, así, asumir un modelo de intervención válido, los programas de intervención, la inconsistencia de los resultados de las investigaciones, la gran variabilidad de técnicas empleadas y la poca sistematicidad aplicada. Esto parece indicar que la prevención y las estrategias de elección son las que reciben más apoyo de los principales autores.

Creo que lo que está de una forma u otra haciendo inviable a la educación son las políticas educativas, legislaciones, presupuestos destinados a educación de forma indistinta y el desgaste por la saturación de actividades que cada día nos alejan más del objetivo de vincular al docente con el alumnado.

\section{BIBLIOGRAFÍA}

1. Anderson MB, Iwanicki EF. Teacher motivation and its relationship to teacher Burnout. Educ Adm Q 1984; 20: 94-132.

2. Atance JC. "Aspectos epidemiológicos del síndrome de Burnout en el personal sanitario". Rev Esp Salud Pública 1997. Disponible en: www. msc.es/epidemiologia/199703/Burnout.html

3. Beer y Beer J. Burnout and stress, depression, and self-esteem of teachers. Psychol Rep 1992; 71: 1331-1336.

4. Brenner, S.O. (1982). Work, health, and well-being for Swedish elementary school teacher. Stress Research Reports, ${ }^{\circ}$ 158. Stockholm: Laboratory for Clinical Stress Research.
5. Burke RJ, Greenglass ER. The client's role in psychological Burnout in teachers and administrators. Psychol Rep 1989; 64: 1299-1306.

6. Byrne BM. Burnout: Investigating the impact of background variables for elementary, intermediate, secondary and university educators. Teaching and teacher education. An International Journal of Research 1991; 7: 197-209.

7. Byrne BM. The nomological network of teacher Burnout: A literature review and empirically validated model. In: Vandenberghe R, Huberman AM (Eds). Understanding and preventing teacher Burnout. Nueva York: Cambridge University Press 1999: 15-37.

8. Calvete E, Villa A. Burnout y síntomas psicológicos: modelo de medida y relaciones estructurales. Ansiedad y Estrés 2000; 6: 117-130.

9. Capel SA. The incidence of and influences on stress and Burnout in secondary school teacher. Br J Educ Psychol 1987; 57: 279-288.

10. Chakravorty B. Mental health among school teachers. In: Cole M, Walker S (Eds). Teaching and stress. Philadelphia: Open University Press 1989: 69-82.

11. Cooke RA, Rousseau DM. Stress and strain from family roles and work role expectation. J Appl Psychol 1984; 69: 252-260.

12. Crane SJ, Iwanicki EF. Perceived role conflict. Role ambiguity and Burnout among special education teachers. Remedial and Special Education 1986; 7: 24-31.

13. De Heus, P. y Diekstra, R.F.W. (1999). Do teachers burn out more easily? A comparison of teacher with other social professions on work stress and burnout symptoms. En R. Vandenberghe y A.M. Huberman (Eds.), Understanding and preventing teacher burnout (pp. 269-284). Nueva York: Cambridge University Press.

14. Doménech B. Introducción al síndrome "Burnout" en profesores y maestros y su abordaje terapéutico. Psicología Educativa 1995; 1: 63-78.

15. Durán MA, Extremera N, Rey L. Burnout en profesionales de la enseñanza: un estudio en educación primaria, secundaria y superior. Psicología del Trabajo y las Organizaciones 2001; 17: 45-62.

16. Esteve (Ed.). Profesores en conflicto. Madrid, Narcea: 23-35.

17. Esteve JM. El malestar docente. $3^{\text {a }}$ ed. Barcelona: Paidós, 1994.

18. Freudenberger HJ, Richelson G. Burnout: The high cost of high achievement. Nueva York: Anchor Press, 1980.

19. García-Calleja M. Bajas por enfermedad. Una investigación crítica Cuadernos de Pedagogía 1991; 251: 80-83.

20. Gil-Monte PR. "Aproximaciones psicosociales y estudios diagnósticos sobre el síndrome de quemarse por el trabajo (Burnout)". Monográfico, Revista de Psicología del Trabajo y de las Organizaciones, 2000.

21. Gil-Monte P, Peiró JM. Desgaste psíquico en el trabajo: el síndrome de quemarse. Madrid: Síntesis, 1997.

22. Gold Y. The factorial validity of the Maslash: Burnout Inventory in a sample of California elementary and junior high school classroom teachers. Educ Psychol Meas 1984; 44: 1009.

23. Gold, Y. y Grant, R.A. (1993). Teachers managing stress and preventing burnout: The professional health solution. Londres: Falmer, 1993.

24. Golembiewski, R.T., Munzenrider, R.F. y Stevenson, J. Stress in organizations. Nueva York: Praeger (1986).

25. Hock RR. . Professional Burnout among public school teachers. Public Pers Manage 1988; 17: 167-189.

26. Keavney G, Sinclair KE. Teacher concerns and teacher anxiety: A neglected topic of classroom research. Rev Educ Res 1978; 48: 273-290.

27. Kelchtermans G, Strittmatter A. Beyond individual Burnout: A perspective for improved schools. Guideline for the prevention of Burnout. In Vandenberghe R, Huberman AM (Eds). Understanding and preventing teacher Burnout. Nueva York: Cambridge University Press, 1999: 304-315.

28. Knight-Wegenstein AG. Die arbeitszeit der Lehrer in der Bundesrepublik Deutschland. Zürich: Autor 1973.

29. Kohnen R, Barth AR. Burnout bei Grund-und-Hauptschullehrern-ein gesundheitliches Risiko? Lehrerjournal Grundschulmagazin 1990; 10: 41-44.

30. Kyriacou C. Stress, health and schoolteachers: A comparison with other professions. Cambridge Journal of Education 1980; 10: 154-158.

31. Kyriacou C. Teacher stress and Burnout: An international review. Educational Research 1987; 29: 146-152. 
32. Kyriacou C. Antiestrés para profesores. Barcelona. Ediciones Octaedro, 2003.

33. Kyriacou C, Sutcliffe J. Teacher stress: A review. Educational Review 1977; 29: 299-306.

34. Kyriacou C, Sutcliffe J. A model of teacher stress. Educ Stud 1978; 4: 1-6.

35. Leithwood KA, Jantzi D, Steinbach R. Maintaining emotional balance. Educational Horizons 2001; 79: 73-82.

36. Malik JL, Mueller RO, Meinke DL. The effects of teaching experience and grade level taught on teacher stress: A LISREL analysis. Teaching \& Teacher Education 1991; 7: 57-62.

37. Maslach C. Burnout: the cost of caring. Englewood Cliffs NJ. Prentice Hall, 1982.

38. Maslach C. Progress in understanding teacher Burnout. In: Vandenberghe R, Huberman AM (Eds). Understanding and preventing teacher Burnout. Nueva York: Cambridge University Press 1999: 211-222).

39. Maslach C, Jackson SE. The measurement of experienced Burnout. Journal of Occupational Behavior 1981; 2: 99-113.

40. Matud MP, García MA, Matud MJ. Estrés laboral y salud en el profesorado: un análisis diferencial en función del género y del tipo de enseñanza. Int J Clin Health Psychol (Revista Internacional de Psicología Clínica y de la Salud)2002; 2: 451-465.

41. McIntyre TC. Faculty development for prior learning programs: The essential ingredients. New Directions for Experiential Learning 1981; 14: 121-132.

42. Moriana. Estudio epidemiológico de la salud mental del profesorado. Córdoba: Servicio de Publicaciones Universidad de Córdoba, 2002.

43. Ortiz VM. Los riesgos de enseñar: la ansiedad de los profesores. Salamanca, Amarú, 1995.

44. Pines AM, Aroson E, Kafry D. Burnout: from tedium to personal growth. Nueva York: Free Press, 1981.
45. Pines AM. Burnout: "An existential perspective". In: Schaufeli WB, Maslach C, Marek T (Eds). Professional Burnout: Recent development in theory and research. Londres: Taylor \& Francis, 1993: 33-51.

46. Sandín B. El estrés: un análisis basado en el papel de los factores sociales. Int J Clin Health Psychol (Revista Internacional de Psicología Clínica y de la Salud) 2003; 3: 141-157.

47. Schwab RL, Iwanicki EF. Perceived role conflict, role ambiguity, and teacher Burnout. Educ Adm Q 1982; 18: 60-74.

48. Schwab RL, Iwanicki EF. Who are our burned out teachers? Educational Research Quarterly 1982; 7: 5-16.

49. Seltzer J, Numerof RE. Supervisory leadership and subordinate Burnout. Acad Manage J 1988; 31: 439-446.

50. Temml Ch. Stre Bim Lehrberuf. Eineösterreichweitestudie 1993. Wien: GÖD. Tomaschevskaja, L.I. (1978). Influence of the working environment and scope of work on the heart-blood circulation system. Kiew: Health, 1994.

51. Travers CT, Cooper CL. Psychophysiological responses to teacher stress: A move towards more objective methodologies. Eur Rev Appl Psychol 1994; 44: 137-146.

52. Travers CT, Cooper CL. El estrés de los profesores. La presión en la actividad docente. Barcelona: Paidós, 1997.

53. Valero L. Comportamientos bajo presión: El Burnout en los educadores. En: Hombrados MI (Ed). Estrés y Salud. Valencia: Promolibro, 1997: 213-237.

54. Van Ginkel AJH. Demotivatiebijleraren. Lisse: Swets y Zeitlinger, 1987.

55. Whitehead JT. Job Burnout and job satisfaction among probation managers. J Crim Justice 1986; 14: 25-35.

56. Yagil D. If anything can go wrong it will: occupational stress among inexperienced teachers. Int J Stress Manag 1998; 5: 179-188. 